\title{
Research and Practice Recommendations for Mixed Reality Design - Different Perspectives from the Community
}

\author{
Veronika Krauß \\ University of Siegen, \\ Verbraucherinformatik Research \\ Group \\ Siegen, Germany \\ veronika.krauss@uni-siegen.de
}

\author{
Florian Jasche \\ University of Siegen, Cyber-Physical \\ Systems \\ Siegen, Germany \\ florian.jasche@uni-siegen.de
}

\author{
Sheree May Saßmannshausen \\ University of Siegen, Institute for \\ Information Systems \\ Siegen, Germany \\ sheree.sassmannshausen@uni- \\ siegen.de
}

\author{
Thomas Ludwig \\ University of Siegen, Cyber-Physical \\ Systems \\ Siegen, Germany \\ thomas.ludwig@uni-siegen.de
}

\author{
Alexander Boden \\ Bonn-Rhein Sieg University of \\ Applied Science, and Fraunhofer FIT \\ Sankt Augustin, Germany \\ alexander.boden@h-brs.de
}

\begin{abstract}
Over the last decades, different kinds of design guides have been created to maintain consistency and usability in interactive system development. However, in the case of spatial applications, practitioners from research and industry either have difficulty finding them or perceive such guides as lacking relevance, practicability, and applicability. This paper presents the current state of scientific research and industry practice by investigating currently used design recommendations for mixed reality (MR) system development. We analyzed and compared 875 design recommendations for MR applications elicited from 89 scientific papers and documentation from six industry practitioners in a literature review. In doing so, we identified differences regarding four key topics: Focus on unique MR design challenges, abstraction regarding devices and ecosystems, level of detail and abstraction of content, and covered topics. Based on that, we contribute to the MR design research by providing three factors for perceived irrelevance and six main implications for design recommendations that are applicable in scientific and industry practice.
\end{abstract}

\section{CCS CONCEPTS}

- Human-centered computing $\rightarrow$ User interface design; Mixed / augmented reality.

\section{KEYWORDS}

Augmented Reality, Guidelines, Design Theory and Practice, Mixed Reality, Design Recommendations, User Interface Design

Permission to make digital or hard copies of all or part of this work for personal or classroom use is granted without fee provided that copies are not made or distributed for profit or commercial advantage and that copies bear this notice and the full citation on the first page. Copyrights for components of this work owned by others than the author(s) must be honored. Abstracting with credit is permitted. To copy otherwise, or republish, to post on servers or to redistribute to lists, requires prior specific permission and/or a fee. Request permissions from permissions@acm.org.

VRST '21, December 8-10, 2021, Osaka, Japan

(c) 2021 Copyright held by the owner/author(s). Publication rights licensed to ACM.

This is the author's version of the work. It is posted here for your personal use. Not

for redistribution. The definitive Version of Record was published in VRST '21,

December 8-10, 2021, Osaka, Japan

ACM ISBN 978-1-4503-9092-7/21/12 ..\$15.00

https://doi.org/10.1145/3489849.3489876

\section{ACM Reference Format:}

Veronika Krauß, Florian Jasche, Sheree May Saßmannshausen, Thomas Ludwig, and Alexander Boden. 2021. Research and Practice Recommendations for Mixed Reality Design - Different Perspectives from the Community. In 27th ACM Symposium on Virtual Reality Software and Technology (VRST '21), December 8-10, 2021, Osaka, Japan. ACM, New York, NY, USA, 13 pages. https://doi.org/10.1145/3489849.3489876

\section{INTRODUCTION}

As the diversity of devices such as smartphones or tablets increased, novel research fields appeared. Emerging spatial technologies like augmented reality (AR), virtual reality (VR), and mixed reality (MR) afford new requirements and interactions while they also include the actual environment as such in the interaction itself. Although spatial technologies have been researched for decades, it was not until the second wave of VR brought new devices to the mass market that research was refueled.

Recent work reports different kinds of design practices and lessons learned for UI design, for instance, in the shape of design principles, guidelines, heuristics, or recommendations $[43,130]$. Those are supposed to support designers in creating usable interfaces with reasonable time and effort [121]. Spatial media research also includes the elicitation and publication of case studies and practices (i.e., [21, 35, 43, 130]).

In this context, current research reports that both researchers and practitioners perceive the present situation in MR development as lacking relevant, practical, and applicable design guides [9, 83], if they are easily accessible at all.

In this paper, we examine the current use and applicability of design recommendations with a particular focus on MR design in practice $[57,83]$. We compare design recommendations for MR systems originating from both scientific research and industry practice to answer these questions:

RQ1 What are the differences between research and practitioner recommendations for AR user interfaces?

RQ2 What factors contribute to them being perceived as relevant or irrelevant for application development?

RQ3 What can we learn from those differences for future work on design recommendations for spatial user interfaces? 


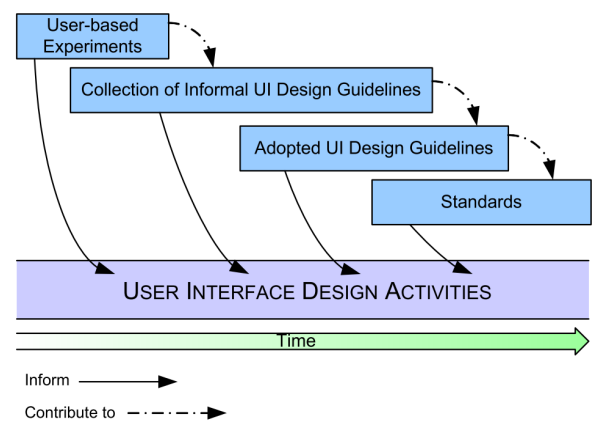

Figure 1: Development of guidelines [49].

In a literature review, we analyzed design recommendations from 89 scientific papers and compared them to those published by Apple, Google, IBM, Magic Leap, Microsoft, and Spark AR. We compared the recommendations regarding four key issues: Focus on MR unique design challenges, abstraction regarding devices and ecosystems, level of detail and abstraction of content, and covered topics. We further formulate-based on our findings-three reasons for the perceived irrelevance of design recommendations for MR and six implications on how to create meaningful design recommendations for both practitioners and researchers.

\subsection{Terms and Concepts in this Paper}

The data set of our analysis contains work that focuses on augmented reality but also includes other types of mixed reality media as defined by the reality-virtuality continuum of Milgram and Kishino [97]. Throughout this paper, we will address the type of media as MR and switch to a more specific differentiation of AR or VR if needed. Furthermore, we differentiate between spatial- and non-spatial media as types of user interfaces (UI). Spatial media consists of UIs that involve spatial components like MR, tangible UIs, or ubiquitous computing. In contrast, non-spatial media encompass more classical UIs, such as command-line interfaces, desktop applications, or systems for mobile devices and tablets following the Windows-Icons-Menus-Pointers (WIMP) paradigm.

\section{RELATED WORK}

\subsection{Designing Interactive Systems}

Among insights from user-based experiments, resources such as informal and adopted design guidelines as well as standards play a central role for designers working to develop usable interactive systems (see Figure 1). In 1976, Cheriton proposed design guidelines for time-shared computer systems with the goal of standardization to "[decrease] the effort required for users to change systems" [26]. Other guidelines exist to provide system designers references to reduce well-known errors because "[most errors] are system induced, a result of inappropriate system design" [103]. Over the last 50 years, the design research field used different terms like design principles, guidelines or heuristics for these proposals. A well-accepted definition for the terms is hard to find. According to Fu et al. [47], these and more terms are part of knowledge explications. Based on a literature review, Fu et al. synthesize the following definitions [47]:

- Principle: A fundamental rule or law, derived inductively from extensive experience and/or empirical evidence, that provides design process guidance to increase the chance of reaching a successful solution.

- Guideline: A context-dependent directive, based on extensive experience and/or empirical evidence, that provides design process direction to increase the chance of reaching a successful solution.

- Heuristic: A context-dependent directive, based on intuition, tacit knowledge, or experiential understanding, that provides design process direction to increase the chance of reaching a satisfactory but not necessarily optimal solution.

Furthermore, design principles are formulated more general to "outlast the technological demands of the moment" [38, 102]. According to Preece et al., guidelines and heuristics are strongly related because design guidelines can be transformed into heuristics for evaluating systems [69]. Without guidelines for specific applications or technologies, designers tend to adapt guidelines from other contexts to new technologies. This bears the risk of neglecting unique features of new technology. User-based experiments help to validate designs and to inform design activities in the early stage of an emerging technology. With further contributions from the community, collections of informal guidelines appear and may evolve into more formal guidelines or standards [49] (see Figure 1). Sometimes, established guidelines are incorporated into design tools that can enforce consistency and ensure reasonable designs of systems [102].

\subsection{Design Recommendations for MR}

$\mathrm{MR}$ is an emerging medium slowly being adapted for mass marketing. As general consent, MR design practices diverge from nonspatial media in presenting and interacting with content. Therefore, this medium requires its own design rules, tools, and practices. Due to its early stage of development, we can observe the evolution of guidelines for designing systems. Endsley et al. [43] investigated 137 statements from AR and related fields and classified them in an iterative process. The result of their work was nine heuristics to be considered by AR designers (see Figure 2). Vi et al. [130] extended Endsley et al.'s work by developing eleven design guidelines for extended reality applications with a focus on head-mounted displays (HMD). In addition to the guidelines from research, they also considered guidelines from the web, including documentation from companies like Google, Leap Motion, or Oculus, as well as blog posts from individuals on platforms like medium.com. The created guidelines are mainly based on online sources and cover both AR and VR. However, due to the popularity of VR at the time of that study, few guidelines were elicited for AR. Both sets bear a likeness to each other (see Figure 2) and to well-known guidelines or heuristics such as Nielsen's 10 usability heuristics [101] for non-spatial interfaces. However, Endsley et al. and Vi et al. provide additional guidelines for $\mathrm{AR}$, such as the three-dimensionality of the medium and a more profound connection of virtuality and reality. Because MR is a body-centric technology, special physical safety and comfort guidelines were added, focusing on ergonomics. 
Concluding our literature review, there were no further attempts to classify existing design recommendations for MR to the best of our knowledge.

\section{RESEARCH APPROACH AND METHODS}

We conducted an iterative literature review oriented on the approach of vom Brocke et al. [22] to answer our research questions (see Section 1). After identifying key concepts and terms, we defined our search terms. As the terms "design guidelines" returned too few results for a proper analysis, we added "design principles" and "design heuristics." The resulting query was: ("Augmented Reality" OR "AR") AND ("design guideline(s)" OR "design principle(s)" OR "heuristic(s)"). We used the Scopus database and searched titles, abstracts, and keywords. Without restricting the period, we received 519 published papers between 2000 and the day of our search, April 17, 2020.

In the first iteration, we removed 34 anthologies and duplicates. The remaining 485 papers were checked for relevance by reading the title, abstract, and conclusion. Papers should mention the design of an AR application or the development of design guidelines or recommendations for AR. As a result, we further excluded papers in which our search terms were used in different contexts (also see Section 1.1) or had a different meaning. We also discarded non-English papers or those that did not have a full-text version publicly available. We kept results stating MR design recommendations instead of AR. Finally, we considered 89 papers for a full-text analysis (see Appendix A). From these papers, we extracted statements that can guide the design of an MR application, such as design principles, guidelines, heuristics, or less formally formulated design recommendations. We will further refer to the extracted information used in our analysis as "statements."

In total, our team of three researchers collected 374 statements on a virtual whiteboard in MURAL and sorted them following an iterative, bottom-up approach into an affinity diagram [64]. We followed an open coding approach described by Strauss and Corbin [4]. Our goal was to elicit common topics regarding design recommendations in MR research. Therefore, we built clusters based on a design recommendation's purpose deduced from each statement. If the purpose was unclear, we clustered regarding a statement's proposed action. Each statement was discussed before we arranged it on the whiteboard. Due to the ambiguity of some design recommendations, the resulting clusters were not mutually exclusive. Hence, we sorted statements that could belong to multiple clusters into the best fitting one based on already arranged statements and our discussions or created a new cluster if none of the arranged statements matched. After all statements had been arranged, we appointed names deduced from a cluster's content before arranging them under matching umbrella topics. We will address those statements and clusters as "Scientific Design Recommendations" (SDRs) in the remaining sections of this paper.

To complement our scientific literature review, we considered design recommendations of companies actively developing MR hardware and software. In October 2020, we queried the websites of six market-leading, AR-related companies Apple [7], Google [58], IBM [66], Magic Leap [94], Microsoft [96], and Spark AR [46]. We extracted design recommendations that we will refer to as "Practitioner Design Recommendations" (PDRs). We analyzed them in line with our approach for SDRs and built clusters by common topics without considering the cluster names from the SDR affinity diagram. Even though some companies already provided a categorization, we followed our bottom-up approach described for SDRs to ensure comparability. This separate affinity diagram has 501 statements from Apple (59), Google (115), IBM (20), Magic Leap (135), Microsoft (103), and Spark AR (68).

\section{DESCRIPTION OF THE DATA SET}

In the following, we describe the features of SDRs and PDRs in detail. Summaries of the affinity diagrams are depicted in Figure 3 for SDRs and Figure 4 for PDRs.

\subsection{Scientific Design Recommendations (SDRs)}

Elicited recommendations either explicitly address hand-held devices ( $n=297 ; 79.4 \%)$, HMDs $(n=42 ; 11.23 \%)$, or did not specify the target device $(n=35 ; 9.36 \%)$. The affinity diagram of 374 statements resulted in seven main topics, which we will further detail. As depicted in Figure 3, each of the main topics consists of several clusters containing mixed statements regarding target devices.

The topic Design Principles (inspired from non-spatial) contains eight clusters and 58 statements (15.51\%) addressing themes known from traditional non-spatial UI design, such as Personalization, Guidance in the sense of tutorials, Learnability in the context of providing manuals, help, and other supportive information to grasp the application, Task appropriateness, Privacy, and Laws such as Hick's Law [62] or the Law of Practice [100]. Furthermore, we grouped statements that vaguely mentioned the adaption of existing guidelines, principles, or heuristics in the cluster Adaption of other heuristics and principles, for example, Shneiderman's design guidelines for desktop application [29, 117] or Nielsen's 10 usability heuristics $[68,128]$ in General Nielsen Heuristics.

We identified 86 statements $(22.99 \%)$ literally quoting or paraphrasing Nielsen's 10 heuristics [68]. Those statements were sorted in the topic Nielsen Heuristics and grouped in the 10 clusters Visibility of system status and feedback, Match between system and the real world, User control and freedom, Consistency and standards, Error prevention, Recognition rather than recall, Flexibility and efficiency of use, Aesthetics and minimalist design, Error recovery, and Help and documentation.

Our SDR data set revealed statements addressing Multi-User Experience, from which we built four clusters with 15 statements (4.01\%). The clusters Collaboration and Social presence in VR are related based on the statements' content. We divided clusters regarding the defined type of media. For instance, "Encourage more communication and interaction during the task" [36] specifically mentioned the design of VR environments and was therefore sorted into the cluster Social presence in VR. In contrast, "Effective tangible $A R$ interfaces can be developed using the design principles learned from tangible user interfaces. The basic principles of TUI include [e.g.] Collaboration between multiple participants" Billinghurst et al. [14] address AR systems and were, therefore, assigned to Collaboration. The cluster Sharing focuses on aspects of sharing interfaces 


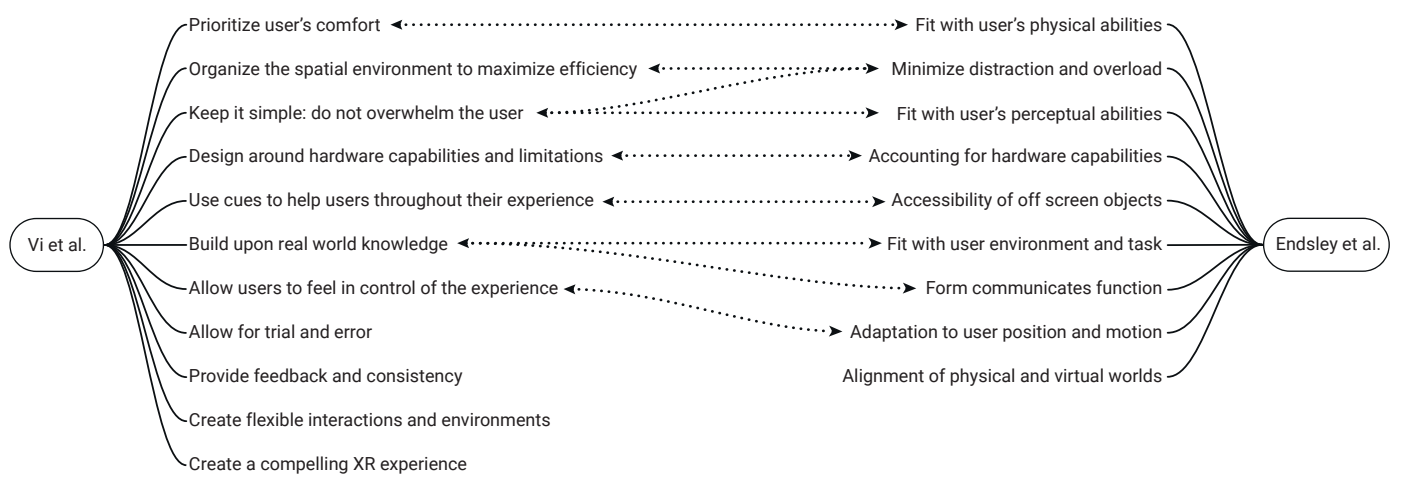

Figure 2: Proposed guidelines and heuristics from Endsley et al. [43] and Vi et al [130]. The dotted lines show similarities between the guidelines.

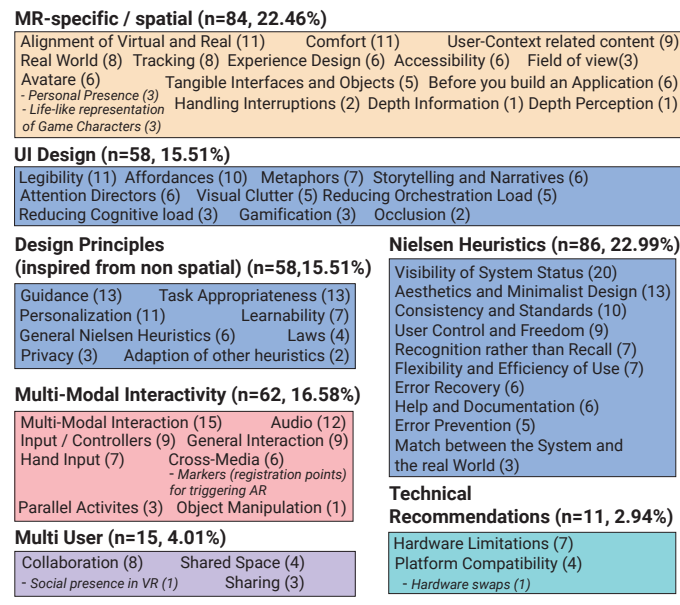

Figure 3: Affinity diagram of scientific design recommendations. Each box represents a topic written as headline that contains multiple clusters. Same-colored boxes are closely related regarding their topics. Cluster names are written inside the boxes, and their sub-clusters are denoted in italics.

and experiences other than Shared spaces, which contains statements about movement, user positioning, and placement of shared content.

In Multi-Modal Interactivity, eight clusters structure 62 statements (16.58\%) focusing on the themes Parallel activities, General interaction like "Creation of appropriate interaction techniques for $A R$ applications that are as intuitive as possible" [72, 142], Multi-modal interaction, Object manipulation, Hand input, Input and controllers, Cross-media, and Audio. The general topic addresses interactivity, either in combination of multiple modalities, spatial interaction, or audio. For instance, Multi-modal interaction combines several modalities like "Gesture-based or verbal speech controls, could also be beneficial" [39].

UI Design addresses more general design recommendations for interface design. Those are closely related to the statements in the topics Design Principles and Nielsen Heuristics. We found
58 statements (15.51\%) and grouped them in 10 clusters. In addition to Occlusion, Attention directors, Gamification, Visual clutter, Affordances, and Metaphors, themes like Reducing cognitive load, Legibility, Storytelling and narratives, and Reducing orchestration load in teaching environments emerged.

The topic Technical Recommendations with three clusters and 11 statements $(2.94 \%)$ concentrates on hardware-induced limitations and cross-device approaches for system development. The clusters are Hardware limitations, Platform compatibility and Hardware swaps.

Finally, the topic MR-specific/Spatial Design consists of 84 statements (22.46\%) grouped into 16 clusters: Field of view, Usercontext related content, Experience design, Handling interruptions, Tangible interfaces and objects, Real world, Life-like representation of game characters, Personal presence, Avatars, Alignment of virtual and real, Tracking, Depth perception, 3D depth information, Comfort, Accessibility, and Before you build an application. This topic is mixed regarding covered themes but is similar regarding the focus on MRapplication-specific features rather than generic recommendations.

\subsection{Practitioner Design Recommendations (PDRs)}

Elicited design recommendations address specific devices, such as handheld (Apple, Google, Spark: $\mathrm{n}=243 ; 45.5 \%$ ) and HMDs (Microsoft, IBM, Magic Leap: $\mathrm{n}=258 ; 51.5 \%$ ). We created 13 main topics for PDRs through affinity diagramming.

In the topic Interactivity, we sorted 67 statements (13.37\%) into 11 clusters. Interaction with objects is often mentioned. We divided these statements into clusters such as user initiated Object placement, Scaling, Rotation, Translation, General manipulation and Visual cues for object manipulation. We found eight statements regarding the Affordance. Statements about rather passive interactions with virtual objects are collected in the cluster Reactive content, like statements about attentive holograms. The cluster Encourage to move also emerged, and we collected four statements about the use of Animations and three statements about the proper introduction of new content to the user in the Content spawn mechanic cluster.

Statements about how the interactivity is implemented are collected in the topic Input Modalities with 68 statements (13.58\%) in 


\begin{tabular}{|c|c|}
\hline \multicolumn{2}{|c|}{ 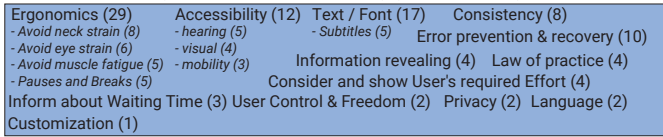 } \\
\hline Guidance $(n=35,6.99 \%)$ & \multirow{2}{*}{$\begin{array}{l}\text { Feedback (n=22, 4.39\%) } \\
\begin{array}{|l}\text { Feedback (7) Audio (6) Notifications (3) } \\
\text { Audio Feedback (3) Haptic Feedback (2) } \\
\text { Immersion (1) }\end{array}\end{array}$} \\
\hline $\begin{array}{l}\text { Attention directors (20) Instructions (10) } \\
\text { Onboarding (5) }\end{array}$ & \\
\hline \multicolumn{2}{|c|}{$\begin{array}{l}\text { Gaze input (15) Selection of Interaction Modality (14) } \\
\text { Hand \& finger gestures (7) Manipulation Gestures (7) } \\
\text { Modality change (3) }\end{array}$} \\
\hline 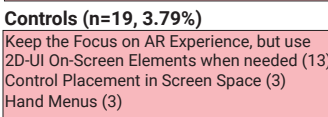 & $\begin{array}{l}\text { Interactivity ( } \mathrm{n}=\mathbf{6 7 , 1 3 . 3 7 \% )} \\
\begin{array}{|l|}\text { Encourage to Move (10) Affordance (8) } \\
\text { Visual Cues for Object Manipulation (8) } \\
\text { Object Scaling (8) Object Placement (8) } \\
\text { Reactive Content (6) Object Manipulation (6) } \\
\text { Animations (4) Content Spawn Mechanic (3) }\end{array}\end{array}$ \\
\hline \multicolumn{2}{|c|}{$\begin{array}{l}\text { Environment ( } n=49,9.78 \%) \\
\begin{array}{l}\text { Space requirements (12) Users' physical safety (12) Space-Robust Applications (9) } \\
\text { Appropriate interplay of virtual and real (10) Transition into AR/VR (6) }\end{array}\end{array}$} \\
\hline Spatial Design $(n=50,9.98 \%)$ & $\begin{array}{l}\text { Technical } \\
\text { Recommendations ( } n=20,3.99 \%)\end{array}$ \\
\hline $\begin{array}{|ll|}\text { Content Placement (15) Field of View (12) } \\
\text { Head-locked Content (9) } & \text { Design Spaces (9) } \\
\text { Anchored UI (5) } & \\
\end{array}$ & \multirow{3}{*}{\begin{tabular}{|l} 
Spatial Anchors (6) Hardware Properties (4) \\
Performance (4) Device support (2) \\
Landscape / Portrait Mode (2) \\
System Architecture (1) \\
Collider for finger gestures (1) \\
\end{tabular}} \\
\hline Detection $(n=25,4.99 \%)$ & \\
\hline \multirow{3}{*}{\begin{tabular}{|l|} 
Surface Detection (8) \\
Handling Relocalization (7) \\
Image detection (4) Spatial mapping (3) \\
Coaching View for Detection (3) \\
\end{tabular}} & \\
\hline & Multi-User Experience $(n=13,2.59 \%)$ \\
\hline & $\begin{array}{l}\text { Multiuser (9) Shared spaces (2) } \\
\text { Social Acceptance (2) }\end{array}$ \\
\hline Realism $(n=26,5.19 \%)$ & Platform Specifics $(n=9,1.80 \%)$ \\
\hline $\begin{array}{|ll|}\text { Visual Appearance of Objects (14) } \\
- \text { - Textures (7) } & \text { Occlusion (6) } \\
-3 D \text { Modeling (4) } & \text { Physics simulation (5) } \\
- \text { Depth Perception (3) } & \text { Avatare (1) }\end{array}$ & $\begin{array}{l}\text { AR Badges and Glyphs (9) Permissions (6) } \\
\text { Magic Leap Styleguide (2) }\end{array}$ \\
\hline
\end{tabular}

Figure 4: Affinity diagram for practitioner design recommendations. Cluster names and topics are denoted as described in Figure 3.

eight clusters. Four of these clusters-Modality change, Gaze input, Voice commands and Cursor-are dominated by statements from Microsoft's Mixed Reality documentation and Magic Leaps' design guides because they address specific design aspects for HMDs. We gathered statements about eye-gaze as well as head-gaze in the cluster Gaze input. We also created a cluster for statements that can guide the Selection of the interaction modality. Statements like "Ensure controls and gestures are ambidextrous" [94] are grouped in the cluster Hand and finger gestures. More specific statements about gestures for object manipulation can be found in the Manipulation gestures cluster. Also closely related to that is the cluster Fitt's Law for touch interaction. Close touch input to manipulable objects should be assumed to be input for the object to facilitate interaction with it.

The topic Environment contains 49 statements (9.78\%) in five clusters focusing on the surrounding space. We created the clusters Space requirements considering the needed space for the experience, Space-robust applications in dynamic environments with content about designing for different physical environments, and Users' physical safety with statements like "Help users move safely in their space. If people are expected to move during the experience, remind them to make space before they make the movement" [46]. Because $\mathrm{AR}$ is embedded in the real world, an Appropriate interplay of virtual content and physical environments as well as the Transition into $A R / V R$ are essential.

We found 26 statements (5.19\%) divided into four clusters focusing on Realism. The biggest cluster is Visual realism and appearance of objects, which contains three sub-clusters about Textures,
$3 D$ modeling and Depth perception. Occlusion is used to provide additional visual feedback and increases the perceived realism. The cluster Physics simulation with statements like "By having your digital objects respond to basic physics in the world, you firmly ground them in reality" [94] is related to the previous cluster. Also connected is the cluster Avatars with one statement from IBM: "Use unrealistic avatars. Realistic avatars can fall into the uncanny valley."

Similar to our SDR data set, we found statements that are related to design principles from non-spatial design. These statements are more general and not directly connected to AR but should also be considered. Overall, we found 95 statements for the topic Design Principles (Inspired from Non-Spatial) that we put into 15 clusters (19.56\%). Because most users are not familiar with AR, Error prevention and recovery is important, and Consistency will increase the learnability. The companies mention different aspects of accessibility when creating an inclusive AR application. For example, there are 12 statements about the legibility of Text/font with five additional statements for Subtitles. We found more statements regarding accessibility

and made clusters for Hearing, Visuals and Mobility. Directly connected to those is the cluster Ergonomics with sub-clusters about avoiding Eye strain, Neck strain and Muscle fatigue as well as including Pauses and breaks. Other clusters in this topic are Consider and show user's required effort, Law of practice, Information revealing, Inform about waiting time, Language, Privacy, User control and freedom and Customization.

Different Detection techniques are used to register digital content into the real environment. We grouped 25 statements $(4.99 \%)$ into five clusters related to detection like Image detection, Surface detection, and more techniques like Spatial mapping. Other statements like "Show users how to find a surface using their phone. Use illustrations or animations to show users how to scan properly" [58] can be found in the Coaching view for detection and Handling interruptions/relocalization clusters.

The topic Guidance collects 35 statements (6.99\%) in three clusters. The biggest cluster is about Attention directors with 20 statements because the combination of the limited field of view and the three-dimensional characteristic of MR makes it necessary to help users find offscreen elements. Another aspect of guidance is Instructions. They should be clear and fit the media, "[f]or example, if you want users to swipe, give them an arrow or a hand icon rather than showing the word 'swipe"' [58]. The last cluster is about Onboarding in an MR application.

We put 13 statements (3.99\%) that mentioned other peopleeither actively participating in the experience or passive bystandersin the topic Multi-user experience with three clusters named Multiuser, Social acceptance and Shared spaces. We also found 20 statements about Technical recommendations. We built the following clusters: Spatial anchors, Performance, Hardware properties, Device support, Landscape/portrait mode, Colliders for finger gestures and System architecture.

22 Statements (4.39\%) like "A sound effect or bump sensation is a great way to confirm that a virtual object has made contact with a physical surface or other virtual object" [7] are part of Feedback. We created a cluster for more universal Feedback statements but also for specific feedback like Haptic feedback for phones and Audio feedback besides Audio in general, Notifications and Immersion. 
Nine statements (1.8\%) are Platform Specific, such as Apples' AR badges and glyphs, Specific Magic Leap style guidelines, and Permissions. Statements about elements to control the flow in the application or to open a menu are collected in the Controls topic. There are 19 statements (3.79\%) in the clusters: Keep the focus on AR experience, but use 2D-UI on-screen elements when needed, Control placement in screen space and Hand menus.

The topic Spatial Design consists of 50 statements (9.98\%) in four clusters. The cluster Content placement includes statements about how to arrange content spatially. Due to technical limitations, the Field of view needs to be considered. The cluster Anchored UI collects statements like "VR and AR experiences should typically attach UI elements to the environment, a tracked controller, or the user's body. 'Anchored' UIs provide higher cognitive ease and require less time to learn." [66], but they have to be separated from statements about Head-locked content because "Implementing 1:1 HUD rotation and translation relative to the user's head motions should always be avoided" [96]. Different spaces like intimate, social and public spaces are considered in the Design space cluster.

\section{SIMILARITIES AND DIFFERENCES BETWEEN SDRS AND PDRS}

Our analysis indicated four dimensions of differences and similarities that we will discuss in detail.

\subsection{Focus on MR Unique Design Challenges}

The SDR data set reflects that it is popular in MR research to apply the design heuristics from Nielsen [101]. As these heuristics originate from usability problems of non-spatial systems in the 90s, an appropriate adaption of their for spatial systems is at least questionable [32]. Nevertheless, statements oriented on either Nielsen's heuristics or other concepts from non-spatial UI design comprise most of the SDR data set. As a result, those proposed recommendations are too generic. Design recommendations should refer to cognitive abilities and human perception [71] and require being unspecific to a certain extent. Therefore, overlap with existing recommendations from classical UI design is likely. However, the statements contained in SDRs appear to be less applicable and relevant in the context of spatial UI design due to their lacking focus on spatial features and issues, such as environment, user orientation, movement, and position in space. Statements considering spatial aspects are also present in SDRs but less dominant than in PDRs.

That becomes more tangible by analyzing clusters of similar topics in SDRs and PDRs, such as ergonomics. As MR systems enable whole-body movement and display spatially distributed information, the physical and mental strain tends to be higher than in non-spatial systems. Therefore, specific recommendations for ergonomic use are indispensable. In SDRs, they are often derived from design principles or heuristics for non-spatial UIs. Those statements remain on a superficial level, for example, "Consider usability and comfort. If a long-term usage is desired, take a comfortable interface for the user into account and consider human factors" [85]. Even more specific formulations are stated without further detail: "Consider the natural viewing angle" [136]. PDRs consider ergonomics from more diverse perspectives, resulting in a larger span of recommendations regarding ergonomic issues caused by spatial activities such as muscle fatigue, eye strain, or neck strain. For instance, Microsoft provides a detailed design recommendation for HMDs: "To avoid eye and neck strain, content should be designed so that excessive eye and neck movements are avoided. Avoid gaze angles more than 10 degrees above the horizon (vertical movement). Avoid gaze angles more than 60 degrees below the horizon (vertical movement). Avoid neck rotations more than 45 degrees off-center (horizontal movement)." In conclusion, SDRs focus less on MR-specific design features and often remain on a superficial level compared to PDRs.

\subsection{Abstraction Regarding Devices and Their Ecosystems}

SDRs are mostly device agnostic and do not emphasize the need to comply with device limitations, hardware properties, or ecosystems. In contrast, several PDR statements guide compatibility with the devices' hardware features, software platforms, and respective ecosystems. For example, Magic Leap published guidelines for dealing with platform limitations and conventions: "Certain control actions must be familiar, intuitive, and adhere to platform conventions." Similarly, Apple's guidelines contain the use of their AR badges and glyphs to trigger the start of an AR experience: "Keep badge placement consistent and clear. A badge looks best when displayed in one corner of an object's photo. Always place it in the same corner and make sure it is large enough to be seen clearly (but not so large that it occludes important detail in the photo)."

Other statements address hardware properties and how to apply them in usable and compelling experiences. Those properties are, for instance, Magic Leap's controller with defined button actions and their use. Similarities exist regarding device-specific handtracking gestures or design recommendations for hand-menus (Microsoft), which are missing in SDRs. Additionally, technical limitations and how to avoid them are essential aspects of PDR. For instance, we found recommendations for solving technical procedures like spatial mapping (Microsoft) or texture resolutions: "To let your scene load faster, don't make textures too large. Their resolution should be $2 k$ at most" [58].

Finally, there are also firm guidelines regarding publication in app stores. One example provides the Magic Leap documentation: "Ensure your immersive app presents a clear exit or quit option when users tap the Home button. In the future, failing to enable this will cause your app to fail the submission process." It seems apparent for companies to explicitly address hardware features and platform conventions to establish standards for their ecosystem. These standards will lower the entry barrier for users and require less effort when learning how to use such applications. Additionally, it makes sense that design recommendations originating from human-computer interaction (HCI) research focus on the medium rather than specific devices because HCI research is not bound to ecosystems or hardware. Therefore, we assume that SDRs are, in general, device agnostic and explorative regarding applied technologies or mixed hardware approaches, whereas PDRs are device- and ecosystemspecific and focus on establishing standards. However, PDRs contain several design recommendations that are neutral regarding hardware, platforms, and ecosystems and hold in a broader context of MR design. Such topics are, for instance, realistic appearance of objects, recreation of real-life physics, designing for dynamic 


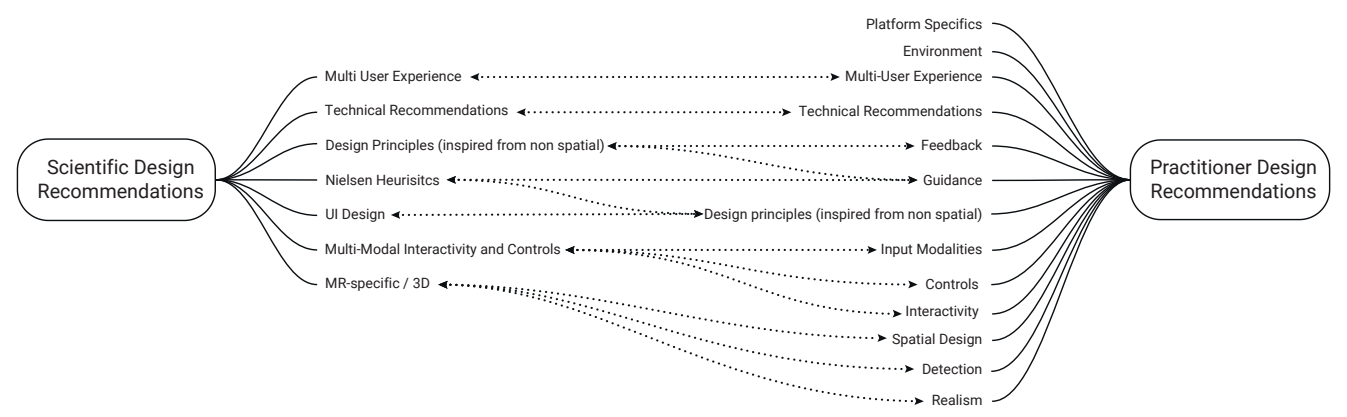

Figure 5: Topics of both SDRs and PDRs and their inter-cluster relationships

environments, and object manipulation. Therefore, we can say that statements in SDRs focus on a subset of PDRs regarding design recommendations.

\subsection{Level of Detail and Abstraction of Content}

Design recommendations require a certain abstraction level to be used in diverse contexts. For example, recommendations might have a too-narrow scope if they are based strictly on layout choices for unique application settings rather than cognitive abilities and limitations. However, design recommendations need to provide context to evaluate their relevance, adapt them to a broad set of UI designs, and allow designers to understand their purpose.

Our analysis reveals the amount of information given with a design recommendation and the type of their abstraction as the main differences between SDRs and PDRs. SDRs tend to be of high abstraction regarding intended use and effect and give few explanations. That requires rereading parts of the statement's source to understand its potential for designing a system. At the same time, that level of abstraction leaves room for interpretation and experimental approaches. For example, Youm et al. formulated six design recommendations through their experience in mobile AR game development, such as: "Provide useful interactions with the AR content: Provide information related to the product or object that empowers the user interaction and experience" [141]. It is unclear what "useful interaction" means, how it affects the experience, and under what circumstances such information should be provided. Hence, this needs to be explored.

That lack of context makes SDRs appear theoretical and difficult to apply without experimenting. However, scientific papers describe detailed contextual information regarding the specific use cases and consider that also in the recommendations, for example, " $A R$ design content should be based on the curriculum, and the time used for AR-based teaching should not be longer than that used to teach the same content with conventional teaching model" [90]. Such context information cannot be found in PDRs.

Furthermore, authors of SDRs rarely provide categories for thematic clustering. Those that do provide thematic clustering often refer to theoretical frameworks, abstract mental models, or ongoing research discourses, such as Ko et al.'s usability grouping system with classifiers like "User-Information", "User-Cognitive", or "User-Interaction" [79]. That categorization abstracts design recommendations from the intended use and effect but supports the identification of existing concepts in $\mathrm{HCI}$ research. In contrast,
PDRs use categories regarding their context or intention from a developer's or a user's perspective, such as Google's AR guidelines with categories like "Environment", "Movement" or "Realism." Furthermore, PDRs abstract from the application context rather than the intended use and effect: "Let the user select a virtual object to identify, manipulate, and interact with it. [...] Use color combinations, glowing outlines, or other visual highlights to let users know. This is especially critical in apps where multiple objects can be selected" [58]. Additionally, PDRs often present concrete solutions for usability issues and include examples and illustrations to facilitate their design recommendations. Consequently, PDRs leave fewer open questions and encourage application rather than experimentation.

We conclude that SDRs mainly foster experimentation through abstracted design recommendations from the intended use and effect. Furthermore, they reflect ongoing research discourses and provide general applicability without giving concrete examples. In contrast, PDRs are formulated from a developer's or user's perspective, abstracted from the application context, and provide examples of intended use and effect. While they usually do not restrict experimentation, they emphasize good practices.

\subsection{Covered Topics}

The topics and clusters emerged from the recommendations' content following a bottom-up principle. The identified topics and clusters can be used in future work as a reference to evaluate new recommendations for semantic similarity and to identify gaps in thematic coverage. In comparison, SDRs and PDRs share similar concepts and topics (see Figure 5) on a divergent level of detail, such as statements regarding general design principles from non-spatial contexts, multi-user experiences, and technical recommendations.

Furthermore, the data sets differ regarding their variance of topics. For instance, we identified MR-specific statements in both data sets dealing with the interplay of virtual and real content, but only a few statements in SDRs address this topic. In contrast, PDRs consider a broader spectrum of issues such as the environment (e.g., space requirements, users' physical safety, dynamic environments, and design spaces), the detection of images and surfaces, visual realism, physics simulations, head-locked content, and general anchored UI elements.

Given the differences regarding the level of completeness and pervasiveness of topics in both data sets, we conclude: SDRs provide a horizontal, framework-like coverage of topics, including experimental areas like tangible AR. PDRs focus on horizontal and vertical 
topic coverage to ensure applicability with a greater level of detail. We argue that this is related to two factors: The first factor is the availability and applicability of mass-market-ready hardware. Hardware is rarely at a high level when research begins to investigate a topic. Therefore, it is challenging to formulate detailed design recommendations beyond interaction paradigms and frameworks. In contrast, PDRs explicitly investigate the applicability of existing hardware in realistic scenarios to minimize the entry hurdle for practitioners. That leads to better coverage of sub-topics and edge cases, including observations from applying technology in the wild, such as dynamic environments, users' physical safety, and accessibility issues. The second factor is that long-term studies in HCI are rare, and evaluations are often part of short-term user studies or lab work $[35,81]$. That leads to relatively small and potentially biased data sets due to the limited time users are given to adapt to new systems. Hence, a topic can only be investigated in-depth if new work focuses on applying existing design recommendations to adapt and refine them. Nevertheless, we found only a few papers experimenting with the adaption of previously defined design recommendations and publishing their results, such as Endsley et al. [43] and de Almeida Pacheco et al. [32]. However, companies can evaluate their existing hardware and applications in realistic settings or draw from a vast set of applications published by the community or created by their developers. That leads to a faster, many-faceted, and consistent, in-depth analysis of potential and applicable design recommendations matching platform and medium-specific topics.

\section{DISCUSSION}

The following discussion addresses our results in the context of our stated research questions. Because we described our findings regarding RQ1 in Section 5, we concentrate on RQ2 in Section 6.1 and RQ3 in Section 6.2. As detailed in Section 5, the main differences between SDRs and PDRs relate to four dimensions: Focus on design challenges unique to $\mathrm{MR}$, abstraction regarding devices and ecosystems, level of detail and abstraction of content, and covered topics. In addition, design recommendations have at least two target groups: researchers and practitioners. Consequently, recommendations serve different needs and purposes and require a clear distinction regarding their intended use and effect.

\subsection{Perceived Relevance and Irrelevance of Design Recommendations}

The issue with design guidelines being perceived as irrelevant has been reported in recent XR-focused work [9] and for design recommendations originating from practice. Beck and Ekbia investigated reasons for the perceived irrelevance of scientific output for practitioners and identified three potential reasons: the problem of communication, abstraction, and research-induced bias [12]. Building on that work, we identified three more factors potentially contributing to perceived relevance or irrelevance for design recommendations in research and practice:

The terms "design principles," "design guidelines," and "design heuristics" are regularly used as synonyms. However, as pointed out in Section 2.1, they are not the same [38, 47, 69, 102]. Hence, published design recommendations add the problem of ambiguous wording, which leads to search results containing design recommendations of varying abstraction levels with different purposes. Based on our findings, the levels of detail and abstraction regarding devices, application, and context are crucial for serving the needs of the target group and therefore add to perceived relevance or irrelevance. Consequently, resolving ambiguous wording requires specifying the target group and the application context, as discussed in the next section.

Design recommendations fail to state their intended use, goals, and target group. Design recommendations address different target groups with different types of information. Whereas scientific recommendations aim for divergence through experimentation, practitioner recommendations provide greater detail for guiding application and aim for convergence [111]. As a result, the latter is perceived of lesser relevance in a scientific context, whereas design recommendations providing superficial guidance require specification effort to be applicable in practical scenarios [20, 55, 65]. It helps to decide if a design recommendation can serve one's needs by explicitly stating the target group and the intended use. Nevertheless, this is insufficient if design recommendations are excluded from search queries due to prejudices against their publication channels, as we will discuss in the next section.

The medium of publication denotes who perceives design recommendations as being relevant. Our SDR data set contains design recommendations targeting practitioners and researchers. However, as we know from literature, practitioners rarely consider scientific databases or attend conferences such as $\mathrm{CHI}[23,123]$. This means that design recommendations for practitioners are most likely not seen if they are published exclusively in academic media due to a biased assumption of irrelevance. Beck and Ekbia also suggest that inaccessibility feeds this prejudice because scientific publications are often hidden behind paywalls [12]. As a consequence, design recommendations for scientific purposes are well-placed on scientific platforms, whereas design recommendations for practitioners are a good fit for respective platforms like Medium, YouTube, and Stack Overflow [9].

\subsection{Towards establishing good design practices}

Creating and defining good design practices require both scientific and practical exploration and application of design recommendations. Based on our study, we would emphasize the following six implications:

Investigate, validate, and adapt existing design recommendations. Ideally, focus on the context of intended use and effect for spatial system development as well as the recommendations' impact on design and user appropriation. The key to providing relevant and applicable design recommendations is their grounding and validation in well-executed user studies combined with a diverse pool of data sets. Hereby, long-term user studies should be preferred to minimize bias, such as the novelty effect [116].

Build recommendations on reliable and transparent data; share experiments and practices. Defining design recommendations requires experience and appropriate data. Hence, recommendations should build on distinct and implemented designs. This could also 
be achieved by considering high-quality data sets published by the AR/VR community, which provide essential metadata regarding design decisions, potential design patterns, good practices, and their evaluation, including the process of requirements elicitation, demographics, study design and procedure, environment, and a detailed application description. Finally, stating how a design recommendation can support a researcher or practitioner and enhance the application's usability and user experience eases assessing a design recommendation's relevance.

Recommendations for research need to foster experimentation and strive for generating knowledge (divergence) [111]. Design recommendations for research should aim to build a thematic framework that could be explored and specified through application, observation, and evaluation. This explorative approach enables researchers to identify several potential solutions, to experience conceptual failures and working solutions and generate knowledge about a design space's specific challenges and rules. Such design recommendations should be grounded in and discussed regarding existing discourse and established concepts, paradigms, and theories. Finally, design recommendations for exploration aim for generalization and abstraction from context, domain, and user intent. This makes them difficult to apply in practice [111], which requires more specific recommendations.

Recommendations for practice need to guide the development of usable systems (convergence) [111]. Design recommendations for practitioners must guide system development, ideally supported by detailed explanations and examples showing the effect of use. Those examples need to enable creators to learn about and understand both the opportunities and limitations of designing for spatial media and its implications for users using the resulting application. Contrasting design recommendations for research, they foster design synthesis [111] and can take hardware specifics into account.

Be clear about intended use and effect, user groups, and wording. Due to the ambiguous use of design principles, guidelines, and heuristics, communicating the intended use and effect of design recommendations is difficult solely based on the wording. While it is desirable to better differentiate between the types of design recommendations for minimizing confusion, it is unlikely that new or enhanced definitions will be adopted likewise from science and research in the near future. Therefore, we suggest including details about the intended usage and context of design recommendations. Following this suggestion, recommendations need to be formulated accordingly to fit the needs of the target group. This includes matching publication and distribution channels of such recommendations.

Aim for a clear distinction of design recommendations and their purpose in research and practice. As we noticed, there is no welladapted definition of design principles, design heuristics, and design guidelines. While existing work describes their derivation and transformation $[69,117]$, the current state of the art lacks a proper differentiation and its adoption. However, providing guidelines for formulating appropriate design recommendations requires dedicated research building on existing work such as [47]. When working towards more distinct definitions, potential factors are (1) intended use and effect, like divergence and convergence and (2) level of abstraction regarding domains, devices, and user intent.

\subsection{Limitations}

We started with investigating AR design recommendations but diverged to VR and finally MR. The mixture of those terms in literature makes it difficult to differentiate our findings because AR and VR are not interchangeable. However, only a few guidelines from SDRs were explicitly created for VR applications. When creating PDRs, we excluded VR-specific guidelines from Google and LeapMotion to ensure comparability. We focused on recommendations of six market-leading AR-related companies. However, there are additional sources for guidelines from practice, for instance, blogs like Medium or less known companies. Including their recommendations warrants a deeper study. In contrast to design guidelines from scientific literature, practitioner design recommendations are constantly being updated. Hence, the design recommendations used in our analysis might have been updated or removed by the time this paper was published. Furthermore, it is known that MR also combines several other disciplines, such as game design and tangible interfaces. We did not perform an explicit search in those areas due to the scope of our research questions. This might exclude potentially applicable design recommendations.

Finally, there might be scientific work that does not focus on formulating design recommendations but still publishes good practices. As a common limitation of literature studies such as ours, those types of publications were not considered because the papers did not contain our search terms.

\section{CONCLUSION}

We investigated the current state of existing design recommendations for MR applications. The literature review examined 89 scientific publications and documentation from companies actively participating in AR and MR development (Apple, Google, IBM, Magic Leap, Microsoft, and Spark AR). In total, we extracted 875 statements from the materials that can meaningfully guide MR application design into two separate data sets based on the source of the statement. We analyzed these data sets through independent affinity diagrams to find common topics in both areas to further investigate different and similar recommendations for MR design. We were able to demonstrate the differences between design recommendations from science and practice. Our findings present insights regarding four key aspects: the focus on design challenges unique to $\mathrm{MR}$, an abstraction regarding devices and ecosystems, the level of detail and abstraction of content, and covered topics. Finally, we deduce six implications for future design recommendation work regarding appropriate adaptions of existing design recommendations, a call for more exploration in MR design, sharing current experiences and practices with the community, appropriate definition of the target group, the intended use of context and formulation of recommendations to guide the development of usable MR systems and a clear distinction of design recommendations and their purpose in design research and practice.

\section{REFERENCES}

[1] Nur Intan Adhani, Dayang Rohaya, and Awang Rambli. 2014. A multimodal interaction design guideline for mobile augmented reality application. 301-306. 
https://doi.org/10.2495/ICACC130411

[2] Mesut Alptekin and Katrin Temmen. 2018. Design concept and prototype for an augmented reality based virtual preparation laboratory training in electrical engineering. In 2018 IEEE Global Engineering Education Conference (EDUCON). IEEE, 963-968. https://doi.org/10.1109/EDUCON.2018.8363334

[3] Marvin Andujar, Anton Nijholt, and Juan E. Gilbert. 2017. Mobile Augmented Games in Playable Cities: Humorous Interaction with Pokémon Go. 575-586. https://doi.org/10.1007/978-3-319-58697-7_43

[4] Anselm Strauss and J. M. Corbin. 1990. Basics of qualitative research: Grounded theory procedures and techniques. Sage Publication. 1-272 pages.

[5] Panagiotis E. Antoniou, Eleni Dafli, and Panagiotis D. Bamidis. 2015. Design of Novel Teaching Episodes in Medical Education Using Emerging Experiential Digital Assets: Technology Enabled Medical Education Beyond the Gimmicky. In 2015 IEEE International Conference on Computer and Information Technology; Ubiquitous Computing and Communications; Dependable, Autonomic and Secure Computing; Pervasive Intelligence and Computing. IEEE, 1560-1565. https: //doi.org/10.1109/CIT/IUCC/DASC/PICOM.2015.360

[6] Panagiotis E. Antoniou, Efstathios A. Sidiropoulos, and Panagiotis D. Bamidis 2017. DISCOVER-ing Beyond OpenSim; Immersive Learning for Carers of the Elderly in the VR/AR era. 189-200. https://doi.org/10.1007/978-3-319-60633$0 \_16$

[7] Apple Inc. [n.d.]. Human Interace Guidelines: Augmented Reality. https://developer.apple.com/design/human-interface-guidelines/ios/systemcapabilities/augmented-reality/

[8] Dawn M. Armfield, Ann Hill Duin, and Isabel Pedersen. 2018. Experiencing Content: Heuristics for Human-Centered Design for Augmented Reality. In 2018 IEEE International Professional Communication Conference (ProComm). IEEE, 241-247. https://doi.org/10.1109/ProComm.2018.00057

[9] Narges Ashtari, Andrea Bunt, Joanna McGrenere, Michael Nebeling, and Parmit K. Chilana. 2020. Creating Augmented and Virtual Reality Applications Current Practices, Challenges, and Opportunities. Conference on Human Factors in Computing Systems - Proceedings (2020), 1-13. https://doi.org/10.1145/ 3313831.3376722

[10] Andrew Aultman, Spencer Dowie, and Nelly Ann Hamid. 2018. Design Heuris tics for Mobile Augmented Reality Game User Interfaces. In Extended Abstract of the 2018 CHI Conference on Human Factors in Computing Systems. ACM, New York, NY, USA, 1-5. https://doi.org/10.1145/3170427.3188580

[11] Zhen Bai, Alan F. Blackwell, and George Coulouris. 2015. Using Augmented Reality to Elicit Pretend Play for Children with Autism. IEEE Transactions on Visualization and Computer Graphics 21, 5 (5 2015), 598-610. https://doi.org/10. 1109/TVCG.2014.2385092

[12] Jordan Beck and Hamid R. Ekbia. 2018. The theory-practice gap as generative metaphor. In Conference on Human Factors in Computing Systems - Proceedings, Vol. 2018-April. Association for Computing Machinery, New York, NY, USA 1-11. https://doi.org/10.1145/3173574.3174194

[13] Mark Billinghurst, Adrian Clark, and Gun Lee. 2015. A Survey of Augmented Reality. Foundations and Trends ${ }^{\circledR}$ in Human-Computer Interaction 8, 2-3 (2015), 73-272. https://doi.org/10.1561/1100000049

[14] Mark Billinghurst, Raphael Grasset, and Julian Looser. 2005. Designing aug mented reality interfaces. ACM SIGGRAPH Computer Graphics 39, 1 (2 2005), 17 https://doi.org/10.1145/1057792.1057803

[15] Mark Billinghurst, Raphael Grasset, and Julian Looser. 2005. Designing aug mented reality interfaces. ACM SIGGRAPH Computer Graphics 39, 1 (2 2005), 17-22. https://doi.org/10.1145/1057792.1057803

[16] Mark Billinghurst, Raphaël Grasset, and Hartmut Seichter. 2010. Tangible Interfaces for Ambient Augmented Reality Applications. In Human-Centric Interfaces for Ambient Intelligence. Elsevier, 281-302. https://doi.org/10.1016/ B978-0-12-374708-2.00011-5

[17] Mark Billinghurst, Hirokazu Kato, and Ivan Poupyrev. 2008. Tangible augmented reality. In ACM SIGGRAPH ASIA 2008 courses on - SIGGRAPH Asia '08. ACM Press, New York, New York, USA, 1-10. https://doi.org/10.1145/1508044.1508051

[18] Timo Bleeker, Gun Lee, and Mark Billinghurst. 2013. Ego- and Exocentric interaction for mobile AR conferencing. In 2013 IEEE International Symposium on Mixed and Augmented Reality (ISMAR). IEEE, 1-6. https://doi.org/10.1109/ ISMAR.2013.6671823

[19] Felix Bork, Alexander Lehner, Daniela Kugelmann, Ulrich Eck, Jens Waschke, and Nassir Navab. 2019. VesARlius: An Augmented Reality System for LargeGroup Co-located Anatomy Learning. In 2019 IEEE International Symposium on Mixed and Augmented Reality Adjunct (ISMAR-Adjunct). IEEE, 122-123. https: //doi.org/10.1109/ISMAR-Adjunct.2019.00-66

[20] John Bowers. 2012. The Logic of Annotated Portfolios: Communicating the Value of 'Research Through Design'. (2012).

[21] Doug A. Bowman, Bernd Fröhlich, Yoshifumi Kitamura, and Wolfgang Stuer zlinger. 2005. New directions in 3D user interfaces. In Proceedings - IEEE Virtual Reality, Vol. 5. 312. https://doi.org/10.20870/ijvr.2006.5.2.2683

[22] Jan vom Brocke, Alexander Simons, Bjoern Niehaves, Bjorn Niehaves, Ka Reimer, Ralf Plattfaut, and Anne Cleven. 2009. Reconstructing the giant: On the importance of rigour in documenting the literature search process. (2009).
[23] Andy Budd. 2013. It's All Academic. https://andybudd.com/archives/2013/02/ its_all_academic

[24] Yoonjeong Cha, Sungu Nam, Mun Yong Yi, Jaeseung Jeong, and Woontack Woo. 2018. Augmented Collaboration in Shared Space Design with Shared Attention and Manipulation. In The 31st Annual ACM Symposium on User Interface Software and Technology Adjunct Proceedings. ACM, New York, NY, USA, 13-15. https: //doi.org/10.1145/3266037.3266086

[25] Alberto Chang, Lourdes Montalvo, and Freddy Paz. 2019. Usability and Playability Heuristics for Augmented Reality Video Games in Smartphones. 19-29. https://doi.org/10.1007/978-3-030-23535-2_2

[26] David R. Cheriton. 1976. Man-machine interface design for timesharing systems. In Proceedings of the annual conference on - ACM 76. ACM Press, New York, New York, USA, 362-366. https://doi.org/10.1145/800191.805617

[27] Enrico Costanza, Andreas Kunz, and Morten Fjeld. 2009. Mixed Reality: A Survey. In Human Machine Interaction: Research Results of the MMI Program, Denis Lalanne and Jürg Kohlas (Eds.). Springer Berlin Heidelberg, Berlin, Heidelberg, 47-68. https://doi.org/10.1007/978-3-642-00437-7_3

[28] Sébastien Cuendet, Quentin Bonnard, Son Do-Lenh, and Pierre Dillenbourg. 2013. Designing augmented reality for the classroom. Computers \& Education 68 (10 2013), 557-569. https://doi.org/10.1016/j.compedu.2013.02.015

[29] Oluwadunsin Dabor, Edward Longford, and Stuart Walker. 2019. Design Guidelines for Augmented Reality User Interface: A Case Study of Simultaneous Interpretation. In 2019 11th Computer Science and Electronic Engineering (CEEC)164. IEEE, Colchester, UK, 164-166. https://doi.org/10.1109/CEEC47804. 2019.8974331

[30] Oluwadunsin Dabor, Edward Longford, and Stuart Walker. 2019. Design Guidelines for Augmented Reality User Interface: A Case Study of Simultaneous Interpretation. In 2019 11th Computer Science and Electronic Engineering (CEEC). IEEE, 164-166. https://doi.org/10.1109/CEEC47804.2019.8974331

[31] Oscar Danielsson, Anna Syberfeldt, Rodney Brewster, and Lihui Wang. 2017. Assessing Instructions in Augmented Reality for Human-robot Collaborative Assembly by Using Demonstrators. Procedia CIRP 63 (2017), 89-94. https: //doi.org/10.1016/j.procir.2017.02.038

[32] Beatriz de Almeida Pacheco, Marcelo Guimarães, Ana Grasielle Correa, and Valeria Farinazzo Martins. 2019. Usability evaluation of learning objects with augmented reality for smartphones: A reinterpretation of nielsen heuristics. In Communications in Computer and Information Science, Vol. 847. Springer Verlag, 214-228. https://doi.org/10.1007/978-3-030-05270-6_16

[33] Beatriz de Almeida Pacheco, Marcelo Guimarães, Ana Grasielle Correa, and Valeria Farinazzo Martins. 2019. Usability Evaluation of Learning Objects with Augmented Reality for Smartphones: A Reinterpretation of Nielsen Heuristics. 214-228. https://doi.org/10.1007/978-3-030-05270-6_16

[34] Richard A. del Rio, Russell J. Branaghan, and Rob Gray. 2016. Design Features of Wearable AR Information Display for Surgery and Anesthesiology. Proceedings of the Human Factors and Ergonomics Society Annual Meeting 60, 1 (9 2016), 571-575. https://doi.org/10.1177/1541931213601131

[35] Arindam Dey, Mark Billinghurst, Robert W Lindeman, and J Edward Swan II. 2018. A Systematic Review of 10 Years of Augmented Reality Usability Studies: 2005 to 2014. Frontiers in Robotics and AI / www.frontiersin.org 1 (2018), 1-28. https://doi.org/10.3389/frobt.2018.00037

[36] Arindam Dey, Hao Chen, Chang Zhuang, Mark Billinghurst, and Robert W. Lindeman. 2018. Effects of Sharing Real-Time Multi-Sensory Heart Rate Feedback in Different Immersive Collaborative Virtual Environments. In 2018 IEEE International Symposium on Mixed and Augmented Reality (ISMAR). IEEE, 165-173. https://doi.org/10.1109/ISMAR.2018.00052

[37] Arindam Dey, Hao Chen, Chang Zhuang, Mark Billinghurst, and Robert W. Lindeman. 2018. Effects of Sharing Real-Time Multi-Sensory Heart Rate Feedback in Different Immersive Collaborative Virtual Environments. In 2018 IEEE International Symposium on Mixed and Augmented Reality (ISMAR). IEEE, 165-173. https://doi.org/10.1109/ISMAR.2018.00052

[38] DIN Deutsches Institut für Normung e.V. 2020. Ergonomie der Mensch-SystemInteraktion - Teil 110: Interaktionsprinzipien. (2020).

[39] Andrew Dolce, Joshua Nasman, and Barbara Cutler. 2012. ARmy: A Study of Multi-User Interaction in Spatially Augmented Games. In 2012 IEEE Computer Society Conference on Computer Vision and Pattern Recognition Workshops. 43-50. https://doi.org/10.1109/CVPRW.2012.6239198

[40] Andrew Dolce, Joshua Nasman, and Barbara Cutler. 2012. ARmy: A study of multi-user interaction in spatially augmented games. In 2012 IEEE Computer Society Conference on Computer Vision and Pattern Recognition Workshops. IEEE, 43-50. https://doi.org/10.1109/CVPRW.2012.6239198

[41] Matt Dunleavy. 2014. Design Principles for Augmented Reality Learning. TechTrends 58, 1 (1 2014), 28-34. https://doi.org/10.1007/s11528-013-0717-2

[42] Ralf Elbert and Tessa Sarnow. 2019. Augmented Reality in Order Picking-Boon and Bane of Information (Over-) Availability. 400-406. https://doi.org/10.1007/ 978-3-030-11051-2_61

[43] Tristan C. Endsley, Kelly A. Sprehn, Ryan M. Brill, Kimberly J. Ryan, Emily C. Vincent, and James M. Martin. 2017. Augmented reality design heuristics: Designing for dynamic interactions. Proceedings of the Human Factors and 
Ergonomics Society 2017-Octob, February 2018 (2017), 2100-2104. https://doi. org/10.1177/1541931213602007

[44] Tristan C. Endsley, Kelly A. Sprehn, Ryan M. Brill, Kimberly J. Ryan, Emily C. Vincent, and James M. Martin. 2017. Augmented Reality Design Heuristics: Designing for Dynamic Interactions. Proceedings of the Human Factors and Ergonomics Society Annual Meeting 61, 1 (9 2017), 2100-2104. https://doi.org/ $10.1177 / 1541931213602007$

[45] Hendrik Engelbrecht and Stephan G. Lukosch. 2018. Viability of Augmented Content for Field Policing. In 2018 IEEE International Symposium on Mixed and Augmented Reality Adjunct (ISMAR-Adjunct). IEEE, 386-389. https://doi.org/10 1109/ISMAR-Adjunct.2018.00111

[46] Facebook. [n.d.]. Ready, set, create. https://go.facebookinc.com/SparkAR Design_Guidelines.html

[47] Katherine K. Fu, Maria C. Yang, and Kristin L. Wood. 2016. Design principles: Literature review, analysis, and future directions. Fournal of Mechanical Design, Transactions of the ASME 138, 10 (2016), 1-13. https://doi.org/10.1115/1.4034105

[48] J.L. Gabbard, J.E. Swan, D. Hix, R.S. Schulman, J. Lucas, and D. Gupta. [n.d.] An empirical user-based study of text drawing styles and outdoor background textures for augmented reality. In IEEE Proceedings. VR 2005. Virtual Reality, 2005. IEEE, 11-317. https://doi.org/10.1109/VR.2005.1492748

[49] Joseph L. Gabbard and J. Edward Swan. 2008. Usability engineering for augmented reality: Employing user-based studies to inform design. IEEE Transactions on Visualization and Computer Graphics 14, 3 (2008), 513-525. https://doi.org/10.1109/TVCG.2008.24

[50] Joseph L. Gabbard, J. Edward Swan, and Deborah Hix. 2006. The Effects of Text Drawing Styles, Background Textures, and Natural Lighting on Text Legibility in Outdoor Augmented Reality. Presence: Teleoperators and Virtual Environments 15, 1 (2 2006), 16-32. https://doi.org/10.1162/pres.2006.15.1.16

[51] Joseph L. Gabbard, J. Edward Swan, Deborah Hix, Si-Jung Kim, and Greg Fitch 2007. Active Text Drawing Styles for Outdoor Augmented Reality: A User-Based Study and Design Implications. In 2007 IEEE Virtual Reality Conference. IEEE, 35-42. https://doi.org/10.1109/VR.2007.352461

[52] Roderick Galantay, Jan Torpus, and Maia Engeli. 2004. "Living-Room" Interactive, Space-Oriented Augmented Reality. ACM Multimedia 2004 - proceedings of the 12th ACM International Conference on Multimedia (2004), 64-71.

[53] Subhashini Ganapathy, Glen J. Anderson, and Igor V. Kozintsev. 2011. Empirical evaluation of augmented information presentation on small form factors - navigation assistant scenario. In 2011 IEEE International Symposium on VR Innovation. IEEE, 75-80. https://doi.org/10.1109/ISVRI.2011.5759606

[54] Miguel Angel Garcia-Ruiz (Ed.). 2017. Games User Research. A K Peters/CRC Press, Boca Raton, FL : Taylor \& Francis, 2016. https://doi.org/10.1201/b21564

[55] William Gaver. 2012. What should we expect from research through design? Conference on Human Factors in Computing Systems - Proceedings (2012), 937-946. https://doi.org/10.1145/2207676.2208538

[56] Brian F. Goldiez, Ali M. Ahmad, and Peter A. Hancock. 2007. Effects of Augmented Reality Display Settings on Human Wayfinding Performance. IEEE Transactions on Systems, Man and Cybernetics, Part C (Applications and Reviews) 37, 5 (9 2007), 839-845. https://doi.org/10.1109/TSMCC.2007.900665

[57] Elizabeth Goodman, Erik Stolterman, and Ron Wakkary. 2011. Understanding Interaction Design Practices. In Proceedings of the International Conference on Human Factors in Computing Systems. Vancouver, Canada, 1061-1070. https: //doi.org/10.1145/1978942.1979100

[58] Google. 2021. Augmented reality design guidelines. https://developers.google. com/ar/design

[59] Adam Greenfeld, Artur Lugmayr, and Wesley Lamont. 2018. Comparative Reality: Measuring User Experience and Emotion in Immersive Virtual Environments. In 2018 IEEE International Conference on Artificial Intelligence and Virtual Reality (AIVR). IEEE, 204-209. https://doi.org/10.1109/AIVR.2018.00048

[60] M. Haller, M. Billinghurst, J. Leithinger, D. Leitner, and T. Seifried. 2005. Coeno. In Proceedings of the 2005 international conference on Augmented tele-existenceICAT '05. ACM Press, New York, New York, USA, 40. https://doi.org/10.1145 1152399.1152408

[61] A. Henrysson, M. Billinghurst, and M. Ollila. 2005. Face to face collaborative AR on mobile phones. In Fourth IEEE and ACM International Symposium on Mixed and Augmented Reality (ISMAR'05). IEEE, 80-89. https://doi.org/10.1109/ ISMAR.2005.32

[62] W.E. Hick. 1952. On the rate of gain of information. Quarterly fournal of Experimental Psychology (1952), 11-26. https://doi.org/10.1016/0022-0965(78) 90002-4

[63] Steve Hinske, Marc Langheinrich, and Matthias Lampe. 2008. Towards guidelines for designing augmented toy environments. In Proceedings of the 7th ACM conference on Designing interactive systems - DIS '08. ACM Press, New York, New York, USA, 78-87. https://doi.org/10.1145/1394445.1394454

[64] Karen Holtzblatt, Jessamyn Burns Wendell, and Shelley Wood. 2005. Rapid Contextual Design: A How-To Guide to Key Techniques for User-Centered Design. Ubiquity 2005, March (3 2005), 3. https://doi.org/10.1145/1066348. 1066325
[65] K Höök and J Löwgren. 2012. Strong concepts: Intermediate-level knowledge in interaction design research. ACM Trans. Comput.-Hum. Interact 19, 23 (2012). https://doi.org/10.1145/2362364.2362371

[66] IBM. [n.d.]. AR/VR meets enterprise - Augmented and virtual reality design guidelines. https:/www.ibm.com/design/v1/language/experience/vrar/

[67] Shafaq Irshad and Dayang Rohaya Awang Rambli. 2016. Design Implications for Quality User eXperience in Mobile Augmented Reality Applications. 1283-1294. https://doi.org/10.1007/978-3-319-24584-3_110

[68] Jakob Nielsen. 1995. 10 Usability Heuristics for User Interface Design. https: //www.nngroup.com/articles/ten-usability-heuristics/

[69] Jennifer Preece, Helen Sharp, and Yvonne Rogers. 2015. Interaction Design: Beyond Human-Computer Interaction, 4th Edition / Wiley (4 ed.). Wiley. https://www.wiley.com/en-us/Interaction+Design\%3A+Beyond+Human+ Computer+Interaction\%2C+4th+Edition-p-9781119088790

[70] Catherine S. Johnson, Shambhavi Mahajan, Mehmet Ordu, Samyukta Sherugar, and Bruce N. Walker. 2016. Will o'the Wisp: Augmented Reality Navigation for Hikers. 365-371. https://doi.org/10.1007/978-3-319-40542-1_60

[71] Jeff Johnson. 2014. Designing with the Mind in Mind, Second Edition: Simple Guide to Understanding User Interface Design Guidelines (2nd ed.). Morgan Kaufmann Publishers Inc., San Francisco, CA, USA.

[72] MCarmen Juan, David Furió, Ignacio Seguí, Noemí Rando, and Juan Cano. 2011. Lessons learnt from an experience with an augmented reality iPhone learning game. http://isites.harvard.edu

[73] M. Carmen Juan, David Furió, Ignacio Seguí, Noemí Rando Aiju, and Juan Cano. 2011. Lessons learnt from an experience with an augmented reality iPhone learning game. In Proceedings of the 8th International Conference on Advances in Computer Entertainment Technology - ACE '11. ACM Press, New York, New York, USA, 1. https://doi.org/10.1145/2071423.2071488

[74] R. S. Kalawsky, K. Hill, A. W. Stedmon, C. A. Cook, and A. Young. 2000. Experimental research into human cognitive processing in an augmented reality environment for embedded training systems. Virtual Reality 5, 1 (3 2000), 39-46. https://doi.org/10.1007/BF01418975

[75] Damla Karagozlu and Fezile Ozdamli. 2017. Student opinions on mobile augmented reality application and developed content in science class. TEM fournal 6, 4 (2017), 660.

[76] Jeremy Kerr and Gillian Lawson. 2020. Augmented Reality in Design Education: Landscape Architecture Studies as AR Experience. International fournal of Art \& Design Education 39, 1 (2 2020), 6-21. https://doi.org/10.1111/jade.12227

[77] Sarah Ketchell, Winyu Chinthammit, and Ulrich Engelke. 2019. Situated Storytelling with SLAM Enabled Augmented Reality. In The 17th International Conference on Virtual-Reality Continuum and its Applications in Industry. ACM, New York, NY, USA, 1-9. https://doi.org/10.1145/3359997.3365681

[78] Matjaž Kljun, Klen Čopič Pucihar, Jason Alexander, Maheshya Weerasinghe, Cuauhtli Campos, Julie Ducasse, Barbara Kopacin, Jens Grubert, Paul Coulton, and Miha Čelar. 2019. Augmentation not Duplication. In Proceedings of the 2019 CHI Conference on Human Factors in Computing Systems. ACM, New York, NY, USA, 1-12. https://doi.org/10.1145/3290605.3300333

[79] Sang Min Ko, Won Suk Chang, and Yong Gu Ji. 2013. Usability Principles for Augmented Reality Applications in a Smartphone Environment. International Journal of Human-Computer Interaction 29, 8 (8 2013), 501-515. https://doi.org/ 10.1080/10447318.2012.722466

[80] Sang Min Ko, Won Suk Chang, and Yong Gu Ji. 2013. Usability Principles for Augmented Reality Applications in a Smartphone Environment. International Journal of Human-Computer Interaction 29, 8 (8 2013), 501-515. https://doi.org/ $10.1080 / 10447318.2012 .722466$

[81] Lisa Koeman. 2020. HCI/UX research: what methods do we use? https: //lisakoeman.nl/blog/hci-ux-research-what-methods-do-we-use/

[82] Panos E. Kourouthanassis, Costas Boletsis, and George Lekakos. 2015. Demystifying the design of mobile augmented reality applications. Multimedia Tools and Applications 74, 3 (2 2015), 1045-1066. https://doi.org/10.1007/s11042-0131710-7

[83] Veronika Krauß, Alexander Boden, Leif Oppermann, and René Reiners. 2021. Current Practices, Challenges, and Design Implications for Collaborative AR/VR Application Development. In CHI '21: Proceedings of the 2021 CHI Conference on Human Factors in Computing Systems. ACM, 1-15. https://doi.org/10.1145/ 3411764.3445335

[84] Max Krichenbauer, Goshiro Yamamoto, Takafumi Taketomi, Christian Sandor, and Hirokazu Kato. 2014. Towards Augmented Reality user interfaces in 3D media production. In 2014 IEEE International Symposium on Mixed and Augmented Reality (ISMAR). IEEE, 23-28. https://doi.org/10.1109/ISMAR.2014.6948405

[85] Philipp Ladwig and Christian Geiger. 2019. A Literature Review on Collaboration in Mixed Reality. Number March. 591-600. https://doi.org/10.1007/978-3-31995678-7_65

[86] Philipp Ladwig and Christian Geiger. 2019. A Literature Review on Collaboration in Mixed Reality. 591-600. https://doi.org/10.1007/978-3-319-95678-7_65

[87] Teemu H. Laine and Hae Jung Suk. 2016. Designing Mobile Augmented Reality Exergames. Games and Culture 11, 5 (7 2016), 548-580. https://doi.org/10.1177/ 
1555412015572006

[88] Gun A. Lee, Gerard J. Kim, and Mark Billinghurst. 2007. Interaction Design for Tangible Augmented Reality Applications. In Emerging Technologies of Augmented Reality. IGI Global, 261-282. https://doi.org/10.4018/978-1-59904066-0.ch013

[89] Gun A. Lee, Jonathan Wong, Hye Sun Park, Jin Sung Choi, Chang Joon Park, and Mark Billinghurst. 2015. User Defined Gestures for Augmented Virtua Mirrors. In Proceedings of the 33rd Annual ACM Conference Extended Abstracts on Human Factors in Computing Systems. ACM, New York, NY, USA, 959-964. https://doi.org/10.1145/2702613.2732747

[90] Li Li. 2018. Application of Augmented Reality Technology in Piano Teaching System Design. Educational Sciences: Theory \& Practice 18, 5 (2018), 1712-1721. https://doi.org/10.12738/estp.2018.5.070

[91] Li Li. 2018. Application of Augmented Reality Technology in Piano Teaching System Design. Educational Sciences: Theory \& Practice (2018). https://doi.org/ 10.12738/estp.2018.5.070

[92] Sha Liang. 2016. Design Principles of Augmented Reality Focusing on the Ageing Population. https://doi.org/10.14236/ewic/HCI2016.2

[93] Yi Lin, Jing Chen, Yue Liu, and Yong-Tian Wang. 2015. User Experience Design of VR-AR Hybrid Mobile Browsing System Based on Mental Model. Chinese Journal of Computers 38, 2 (2015), 408-422.

[94] Magic Leap. [n.d.]. Developer Portal. https://developer.magicleap.com/enus/home

[95] Masyarah Zulhaida Masmuzidin and Norazah Abdul Aziz. 2019. The Adaptation of Shneiderman's Golden Rules and Nielsen's Heuristics on Motivationa Augmented Reality Technology Design for Young Children. In 2019 IEEE 9th International Conference on System Engineering and Technology (ICSET). IEEE, 62-67. https://doi.org/10.1109/ICSEngT.2019.8906373

[96] Microsoft. [n.d.]. Mixed Reality-Dokumentation. https://docs.microsoft.com/dede/windows/mixed-reality/

[97] Paul Milgram and Fumio Kishino. 1994. Taxonomy of mixed reality visual displays. IEICE Transactions on Information and Systems E77-D, 12 (1994), 13211329.

[98] Antoni Moore. [n.d.]. A Tangible Augmented Reality Interface to Tiled Street Maps and its Usability Testing. In Progress in Spatial Data Handling. Springer Berlin Heidelberg, 511-528. https://doi.org/10.1007/3-540-35589-8_33

[99] Vijayakumar Nanjappan, Rongkai Shi, Hai-Ning Liang, Haoru Xiao, Kim King-Tong Lau, and Khalad Hasan. 2019. Design of Interactions for Hand held Augmented Reality Devices Using Wearable Smart Textiles: Findings from a User Elicitation Study. Applied Sciences 9, 15 (8 2019), 3177. https //doi.org/10.3390/app9153177

[100] A Newell and P S Rosenbloom. 1982. Mechanisms of skill acquisition and the law of practice. November (1982).

[101] Jakob Nielsen. 1994. Enhancing the explanatory power of usability heuristics Conference on Human Factors in Computing Systems - Proceedings (1994), 152-158. https://doi.org/10.1145/191666.191729

[102] Donald A. Norman. 1983. Design principles for human-computer interfaces. Conference on Human Factors in Computing Systems - Proceedings December (1983), 1-10. https://doi.org/10.1145/800045.801571

[103] Donald A. Norman. 1983. Design Rules Based on Analyses of Human Error Commun. ACM 26, 4 (1983), 254-258. https://doi.org/10.1145/2163.358092

[104] Ardee Joy T. Ocampo. 2019. TourMAR. In Proceedings of the 2019 4th International Conference on Multimedia Systems and Signal Processing - ICMSSP 2019. ACM Press, New York, New York, USA, 79-83. https://doi.org/10.1145/3330393. 3330428

[105] Patrick O'Shea, Rebecca Mitchell, Catherine Johnston, and Chris Dede. 2009 Lessons Learned about Designing Augmented Realities. International fournal of Gaming and Computer-Mediated Simulations 1, 1 (1 2009), 1-15. https: //doi.org/10.4018/jgcms.2009010101

[106] Eric G. Poitras, Jason M. Harley, Timothy Compeau, Kevin Kee, and Susanne P. Lajoie. [n.d.]. Augmented Reality in Informal Learning Settings. In Virtual and Augmented Reality. IGI Global, 804-825. https://doi.org/10.4018/978-1-52255469-1.ch039

[107] Iulian Radu. 2014. Augmented reality in education: a meta-review and crossmedia analysis. Personal and Ubiquitous Computing 18, 6 (8 2014), 1533-1543. https://doi.org/10.1007/s00779-013-0747-y

[108] Iulian Radu, Blair MacIntyre, and Stella Lourenco. 2016. Comparing Children's Crosshair and Finger Interactions in Handheld Augmented Reality. In Proceed ings of the The 15th International Conference on Interaction Design and Children ACM, New York, NY, USA, 288-298. https://doi.org/10.1145/2930674.2930726

[109] Dayang Rohaya Awang Rambli and Shafaq Irshad. 2015. UX design evaluation of mobile augmented reality marketing products and services for Asia Pacific region. In Proceedings of the Asia Pacific HCI and UX Design Symposium. ACM, New York, NY, USA, 42-45. https://doi.org/10.1145/2846439.2846450

[110] Gang Ren, Side Wei, Eamonn O'Neill, and Fenfang Chen. 2018. Towards the Design of Effective Haptic and Audio Displays for Augmented Reality and Mixed Reality Applications. Advances in Multimedia 2018 (7 2018), 1-11. https: //doi.org/10.1155/2018/4517150
[111] David Roedl and Erik Stolterman. 2013. Design Research at CHI and its Applicability to Design Practice.

[112] Cledja Rolim, Dieter Schmalstieg, Denis Kalkofen, and Veronica Teichrieb. 2015. [POSTER] Design Guidelines for Generating Augmented Reality Instructions. In 2015 IEEE International Symposium on Mixed and Augmented Reality. IEEE, 120-123. https://doi.org/10.1109/ISMAR.2015.36

[113] Carlos Santos, Brunelli Miranda, Tiago Araujo, Nikolas Carneiro, Anderson Marques, Marcelle Mota, Jefferson Morais, and Bianchi Meiguins. 2016. Guidelines for Graphical User Interface Design in Mobile Augmented Reality Applications. 71-80. https://doi.org/10.1007/978-3-319-39907-2_7

[114] Marc Ericson C Santos, Takafumi Taketomi, Goshiro Yamamoto, Ma Mercedes T Rodrigo, Christian Sandor, and Hirokazu Kato. 2015. Toward guidelines for designing handheld augmented reality in learning support. In Proceedings of the 23rd International Conference on Computers in Education. Citeseer, 723-728.

[115] Ralph Schoenfelder and Dieter Schmalstieg. 2008. Augmented Reality for Industrial Building Acceptance. In 2008 IEEE Virtual Reality Conference. IEEE, 83-90. https://doi.org/10.1109/VR.2008.4480755

[116] J. Schomaker and M. Meeter. 2015. Short- and long-lasting consequences of novelty, deviance and surprise on brain and cognition. Neuroscience \& Biobehavioral Reviews 55 (8 2015), 268-279. https://doi.org/10.1016/j.neubiorev.2015.05.002

[117] Ben Shneiderman, Catherine Plaisant, Maxine Cohen, Steven Jacobs, and Niklas Elmqvist. 2016. Designing the User Interface: Strategies for Effective HumanComputer Interaction (6 ed.). Pearson. 1-580 pages.

[118] Saidatul A'isyah Ahmad Shukri, Haslina Arshad, and Rimaniza Zainal Abidin. 2017. Mobile Augmented Reality System Design Guidelines Based on Tourist's Emotional State. Fournal of Telecommunication, Electronic and Computer Engineering (fTEC) 9, 2-12 (2017), 75-79.

[119] Carl Smith. [n.d.]. (Re)Engineering Cultural Heritage Contexts using Creative Human Computer Interaction Techniques and Mixed Reality Methodologies. In Hospitality, Travel, and Tourism. IGI Global, 1489-1499. https://doi.org/10.4018/ 978-1-4666-6543-9.ch086

[120] Carl Smith. 2010. The Design and Evaluation of Augmented Learning Spaces.. In EC-TEL Doctoral Consortium. Citeseer, 91-96.

[121] Sidney L. Smith and Jane N. Mosier. 1986. Guidelines for designing user interface software. Technical Report. The MITRE Coorporation, Bedford, MA. 1-329 pages. https://rauterberg.employee.id.tue.nl/lecturenotes/DA308/MITRE(1986) smith-mosier.pdf

[122] Peter Sommerauer and Oliver Müller. 2014. Augmented reality in informal learning environments: A field experiment in a mathematics exhibition. Computers \& Education 79 (10 2014), 59-68. https://doi.org/10.1016/j.compedu.2014.07.013

[123] Vicky Teinaki. 2013. The Naughty and Nice of UX Conferences. https: //www.uxmas.com/2013/the-naughty-and-nice-of-ux-conferences/

[124] Jos P Thalen and M C van der Voort. 2011. User Centred Methods for Gathering VR Design Tool Requirements. In foint Virtual Reality Conference of EGVE EuroVR, Sabine Coquillart, Anthony Steed, and Greg Welch (Eds.). The Eurographics Association. https://doi.org/10.2312/EGVE/JVRC11/075-081

[125] Marina Tomara and Dimitris Gouscos. 2019. A Case Study: Visualizing Coulomb Forces With the Aid of Augmented Reality. Fournal of Educational Computing Research 57, 7 (12 2019), 1626-1642. https://doi.org/10.1177/0735633119854023

[126] Stefano Triberti, Eleonora Brivio, and Carlo Galimberti. 2018. On Social Presence. 20-41. https://doi.org/10.4018/978-1-5225-3229-3.ch002

[127] Tsai-Hsuan Tsai, Hsien-Tsung Chang, Ming-Chun Yu, Huan-Ting Chen, ChunYi Kuo, and Wei-Hung Wu. 2016. Design of a Mobile Augmented Reality Application: An Example of Demonstrated Usability. 198-205. https: //doi.org/10.1007/978-3-319-40244-4_19

[128] Tsai Hsuan Tsai, Ching Yen Shen, Zhi Sheng Lin, Huei Ru Liu, and Wen Ko Chiou. 2017. Exploring location-based augmented reality experience in museums. In Lecture Notes in Computer Science (including subseries Lecture Notes in Artificial Intelligence and Lecture Notes in Bioinformatics), Vol. 10278 LNCS. Springer Verlag, 199-209. https://doi.org/10.1007/978-3-319-58703-5_15

[129] Tsai-Hsuan Tsai, Ching-Yen Shen, Zhi-Sheng Lin, Huei-Ru Liu, and Wen-Ko Chiou. 2017. Exploring Location-Based Augmented Reality Experience in $\mathrm{Mu}-$ seums. 199-209. https://doi.org/10.1007/978-3-319-58703-5_15

[130] Steven Vi, Tiago Silva da Silva, and Frank Maurer. 2019. User Experience Guidelines for Designing HMD Extended Reality Applications. Vol. 11749. 319-341. https://doi.org/10.1007/978-3-030-29390-1_18

[131] Steven Vi, Tiago Silva da Silva, and Frank Maurer. 2019. User Experience Guidelines for Designing HMD Extended Reality Applications. 319-341. https: //doi.org/10.1007/978-3-030-29390-1_18

[132] Philipp Wacker, Oliver Nowak, Simon Voelker, and Jan Borchers. 2019. ARPen. In Proceedings of the 2019 CHI Conference on Human Factors in Computing Systems. ACM, New York, NY, USA, 1-12. https://doi.org/10.1145/3290605.3300849

[133] Daniel Wagner, Dieter Schmalstieg, and Mark Billinghurst. 2006. Handheld AR for Collaborative Edutainment. 85-96. https://doi.org/10.1007/11941354_10

[134] Richard Wetzel, Rod McCall, Anne-Kathrin Braun, and Wolfgang Broll. 2008. Guidelines for designing augmented reality games. In Proceedings of the 2008 Conference on Future Play Research, Play, Share - Future Play '08. ACM Press, New York, New York, USA, 173. https://doi.org/10.1145/1496984.1497013 
[135] Pierre Wijdenes, David Borkenhagen, Julie Babione, Irene Ma, and Greg Hallihan 2018. Leveraging Augmented Reality Training Tool for Medical Education. In Ex tended Abstracts of the 2018 CHI Conference on Human Factors in Computing Systems. ACM, New York, NY, USA, 1-7. https://doi.org/10.1145/3170427.3174361

[136] Yan Xu, Evan Barba, Iulian Radu, Maribeth Gandy, Richard Shemaka, Brian Schrank, Blair Macintyre, and Tony Tseng. 2011. Pre-Patterns for Designing Embodied Interactions in Handheld Augmented Reality Games. In IEEE International Symposium on Mixed and Augmented Reality 2011 Arts, Media, \& Humanities Proceedings. 19-28. www.argamestudio.org

[137] Yan Xu, Evan Barba, Iulian Radu, Maribeth Gandy, Richard Shemaka, Brian Schrank, Blair MacIntyre, and Tony Tseng. 2011. Pre-patterns for designing embodied interactions in handheld augmented reality games. In 2011 IEEE International Symposium on Mixed and Augmented Reality - Arts, Media, and Humanities. IEEE, 19-28. https://doi.org/10.1109/ISMAR-AMH.2011.6093652

[138] Shys-Fan Yang-Mao, Ming-Hui Lin, Yu-Ting Lin, Wen-Jun Zeng, and Yueh-Y Lai. 2013. Exploring Psychophysical Factors Influencing Visibility of Virtual Image Display. 327-335. https://doi.org/10.1007/978-3-642-39191-0_37

[139] Chris Yoon, Ryan Louie, Jeremy Ryan, MinhKhang Vu, Hyegi Bang, William Derksen, and Paul Ruvolo. 2019. Leveraging Augmented Reality to Create Apps for People with Visual Disabilities. In The 21st International ACM SIGACCESS Conference on Computers and Accessibility. ACM, New York, NY, USA, 210-221. https://doi.org/10.1145/3308561.3353788

[140] DongHyun Youm, SangHyun Seo, and Jung-Yoon Kim. 2019. Design and development methodologies of Kkongalmon, a location-based augmented reality game using mobile geographic information. EURASIP fournal on Image and Video Processing 2019, 1 (12 2019), 1. https://doi.org/10.1186/s13640-018-0395-2

[141] Dong Hyun Youm, Sang Hyun Seo, and Jung Yoon Kim. 2019. Design and development methodologies of Kkongalmon, a location-based augmented reality game using mobile geographic information. Eurasip fournal on Image and Video Processing 2019, 1 (2019), 1-12. https://doi.org/10.1186/s13640-018-0395-2

[142] Feng Zhou, Henry Been-Lim Duh, and Mark Billinghurst. 2008. Trends in Augmented Reality Tracking, Interaction and Display: A Review of Ten Years of ISMAR. In IEEE International Symposium on Mixed and Augmented Reality 2008. IEEE, Cambridge, UK, 193-202.

\section{A REFERENCES OF THE LITERATURE REVIEW}

Below, we list the work used to elicit design recommendations from scientific papers contained in SDRs:

$[1-3,5,6,8,10,11,13,15-19,24,25,27,28,30,31,33,34,37,40-$ $42,44,45,48,50-54,56,59-61,63,67,70,73-78,80,82,84,86-89,91-$ 93, 95, 98, 99, 104-110, 112-115, 118-120, 122, 124-127, 129, 131$135,137-140]$ 